\title{
Evaluation of Mural Series on the Evolution of Life
}

\author{
Joana Ricou, Danielle Commisso, Laura Gonzalez, John A. Pollock \\ Duquesne University, USA
}

\begin{abstract}
Besides being heavily politicized, evolution presents a challenge to teachers because major components of the topic remain under public debate and scientific discovery. We present the Spiral of Life series, an art and science collaboration designed to visually communicate both basic and cutting-edge concepts surrounding the phylogeny of life, while attracting and engaging a broad audience. Five custom art pieces based on a shared spiral symbol were developed and installed in Pittsburgh museums, each exploring a perspective on phylogeny complementary to the institution's focus. Begun in Spring 2010, the Spiral is being studied at the host museums and in the classroom to evaluate its teaching effectiveness and the impact of its artistic vision.
\end{abstract}

\section{Introduction}

A Gallup survey in 2007 determined that $41 \%$ of Americans believe that creationism is true and evolution is false, while only $28 \%$ believe the opposite [1]. The survey shows a negative relationship between education and belief in creationism, while revealing a surprising statistic: half of the least schooled subjects had "no opinion" regarding evolution versus creationism. This data suggests there may be a significant younger and undecided audience open to being educated about the fundamental principles of evolution. But even among the $28 \%$ of Americans that do acknowledge evolution, a chasm is felt between the frontier of the science and its popularized conception. These factors motivate the development of new teaching tools that are attractive and accessible to a younger audience, but versatile enough to incorporate new research and demonstrate our changing understanding of evolution.

Charles Darwin introduced the tree of life paradigm in 1837 [2], codifying the ideas that all species are related by common descent and that they diversified. This "tree of life" remains the most common symbol and mental framework for evolution today. The popularization of this revolutionary image has led to some misconceptions: that evolution is a linear, vertical process that started from a single point, and that evolution culminated in the human species and stopped in the modern day. It is hard to assess the negative impact of these misconceptions on issues like public health and policy.

As biological research progresses, an incredibly detailed amount of information about evolution is being generated and new tools are being developed to provide access to this data, like the on-line encyclopedia "Tree of Life web project" [3] and the "Interactive Tree of Life" [4]. iTOL is one of the few visualizations that deviates from the tree image, using the emerging circle theme and showing unrooted trees with the origin in the middle. However, these powerful tools may be less accessible to younger students and beginners in the field.

\section{The Spiral of Life}

To provide public access to both fundamental and cutting-edge principles of science that are central to evolution, we created the Spiral of Life symbol (see Figure 1) [5] and led a partnership between Duquesne University and major cultural institutions in Pittsburgh, including the Carnegie Science Center, Phipps Conservatory \& Botanical Gardens, Pittsburgh Zoo \& PPG Aquarium, The National Aviary, and Children's Museum of Pittsburgh.

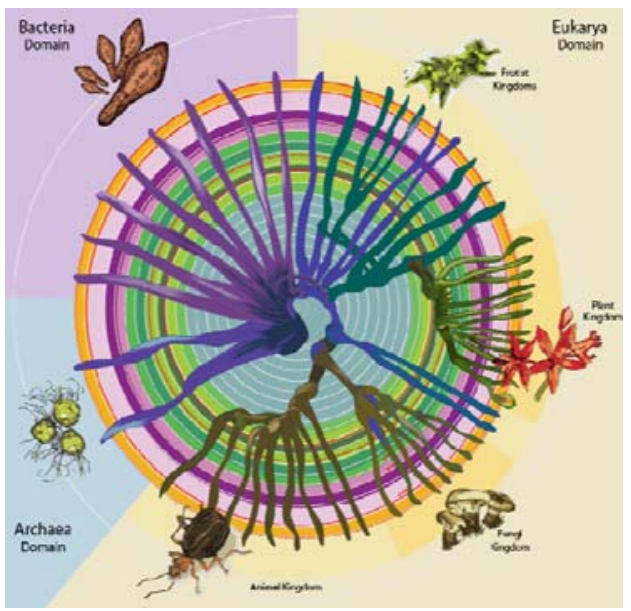

Figure 1. The Spiral of Life symbol 
For each partner museum, we created a distinct but related Spiral art piece [6] (see Figure 2). A scientific advisory board was assembled to discuss and vet the science and to provide feedback on the visualization.

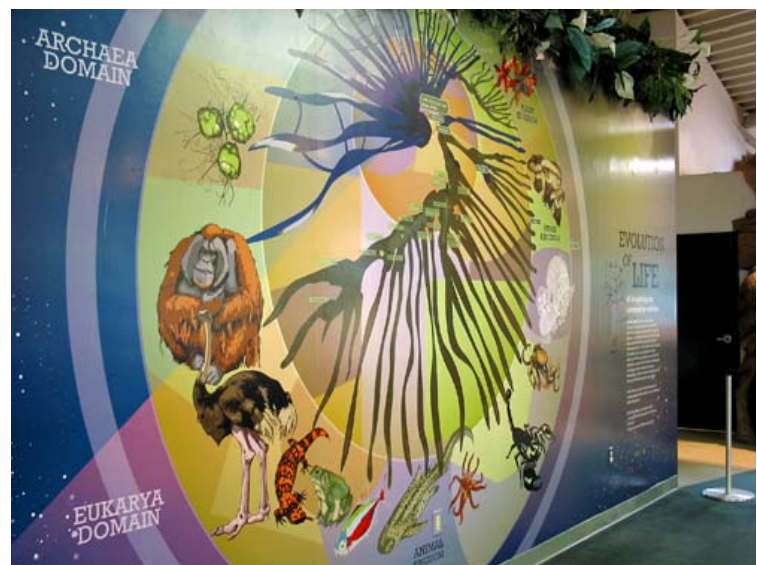

Figure 2. The flexibility of the Spiral permits telling different stories, like the evolution of animals at a zoo.

The key principles to be communicated are as follows.

1) All livings things share a common ancestor. The Spiral combines the ramification pattern seen in Darwin's iconic tree with the circle theme, thus placing the origin at the center of the diagram, unequivocally communicating the common origin of life. The Spiral further innovates visually by representing the origin of life not as a neat single line, but as a tumultuous jumble of building blocks of life, implying that the origin of the three domains (Bacteria, Archaea, and Eukarya) may be more primitive or more varied than previously thought [7].

2) All livings things are equally important, and have their own evolutionary history. The radius of the circle signifies time, like rings in a tree. This visualization helps equate living organisms at each given moment.

3) Evolution is a history of events. The labeling of time helps viewers order major evolutionary events.

4) Evolution is on-going. A "future" ring tantalizes the imagination of the viewer, demanding that they project how branches will evolve.

5) All living things evolve. The colorful and large depiction of representative organisms of each branch is given more importance than its name, stretching the viewers' conception of the diversity of life without the barrier of scientific nomenclature.

6) Evolution is not linear (or vertical). Processes represented in a traditional tree of life are referred to as "vertical," showing the passage of genetic material from a parent generation to an offspring generation. But in the late 20th century, researchers discovered that genetic material can also be passed between organisms of the same generation without reproduction. This process, called "horizontal gene transfer," is expressed in the Spiral, showing that evolution is best understood as a pattern that combines vertical branching and horizontal crossing.

\section{Evaluation}

The evaluation plan assessed attitudes, opinions and knowledge gained through interaction with the Spirals. The study used an unsupervised, interactive survey implemented with a computer touch screen installed at the Pittsburgh Zoo \& PPG Aquarium, at the Carnegie Science Center, and at The National Aviary. The surveys had a common segment that evaluates the symbol and a section that targets specific details. A total of 7,891 surveys were initiated with an overall completion rate of $65 \%$ or 5,290 surveys completed. Our results show that the lack of supervision carries a significant challenge. The opened-ended comments of some of the participants and direct observations by a staff member indicated that they were not necessarily aware that (a) the activity on the touch screen was a survey or (b) that it was about the mural. For example, the following statement "Thank you for making a wonderful zoo!” strongly suggests (b). Subsequent installations of the touch screen survey included different sets of instructions and signage in the survey itself and around the touch screen display.

\section{Results}

The surveys indicate that $70 \%$ of patrons $(\mathrm{n}=3476)$ reported they were 'satisfied' to 'very satisfied' with their level of enjoyment, learning, and information found in the murals.

The survey allowed for the study of several dimensions of the patrons' experience with the mural.

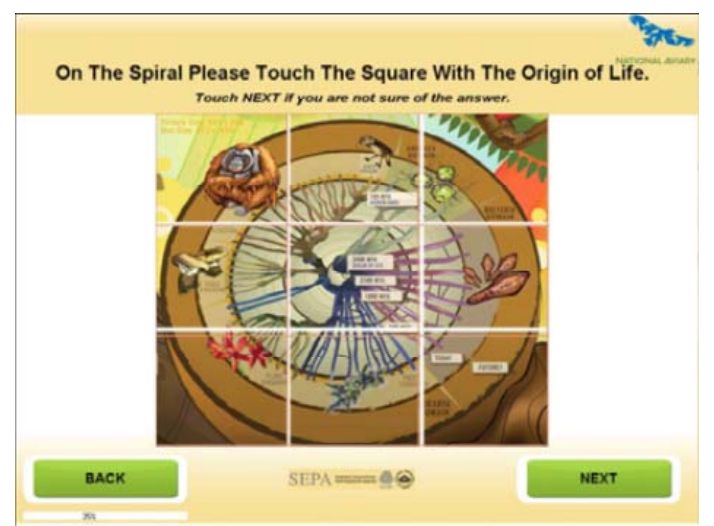

Figure 3. Diagrams were broken out into a $3 \times 3$ grid.

For example, the results to the question "On the Spiral, please choose the Square with the origin of 
Life” were intriguing. This question asked the participants to identify the location of the origin of life in the diagram. On the screen of the question, there was an image of the actual display split into a 3x3 grid (see Figure 3). The participants answered by touching the appropriate block, without an option to skip.

Table 1. Results for the question “... Please choose the square with the origin of life.” The correct answer is in bold

\begin{tabular}{|c|c|c|c|}
\hline $\begin{array}{c}\text { Selected } \\
\text { Square }\end{array}$ & Aviary & $\begin{array}{c}\text { Carnegie } \\
\text { Science } \\
\text { Center }\end{array}$ & $\begin{array}{c}\text { Pittsburgh } \\
\text { Zoo \& PPG } \\
\text { Aquarium }\end{array}$ \\
\hline middle top & $3.70 \%$ & $\mathbf{2 9 . 6 3 \%}$ & $\mathbf{2 4 . 3 0 \%}$ \\
\hline center & $\mathbf{6 0 . 3 0 \%}$ & $18 \%$ & $20.70 \%$ \\
\hline top left & $8.80 \%$ & $20 \%$ & $18.60 \%$ \\
\hline $\mathrm{n}$ & 976 & 1080 & 3584 \\
\hline
\end{tabular}

The success rate on this question is distinctly lower at the science center and at the zoo as compared to the aviary (see Table 1). Spatial orientation of the participant's incorrect answers among the nine choices suggests that they may be primed to assume that the beginning of a diagram will be found on the top-left or center. This may be along the same lines of the documented bias where people tend to read time horizontally in the direction that their language is written in [8]. This indicated that at least some patrons lack flexibility to interpret a new scheme even when labeled, or were overwhelmed by the wealth of information in the image.

Other aspects of the survey assessed whether patrons could identify the correct junction points as representing last common ancestors between two groups and found that at one of the institutions, only $19 \%$ ( $n=1515)$ were correct. This result can mean many things: (a) that participants had no idea what "common ancestor" meant or (b) how that concept relates to an evolutionary diagram, or (c) they couldn't interpret the Spiral. An interesting counter point was that $48 \%$ were able to correctly identify where humans evolved, even though humans were not explicitly marked on the Spiral branch for mammals.

Our analysis suggests that visitors appeared interested in the murals but were struggling to decipher the image. In response to this finding, we produced hand-outs for each museum that verbally summarized the main content points of each display and connected to the imagery [9].

Creating informal science educational materials requires a balance between providing new and familiar information. In the case of the Animal Evolution display, we wrestled with the fact that some animal groups are significantly better known and "popular" (i.e., placental mammals) than others. In consultation with the zoo staff, we decided to include all phylum level groups but also reveal Vertebrate and Arthropods to the class level. To facilitate the interpretation of the mural, a volunteerled activity was created where visitors pick an animal to draw that may or may not be present in the mural and, with guidance, figure out a location for that animal in the diagram (Figure 4). Drawings were added to the mural across most branches of the Spiral with an emphasis on those that had illustrations.

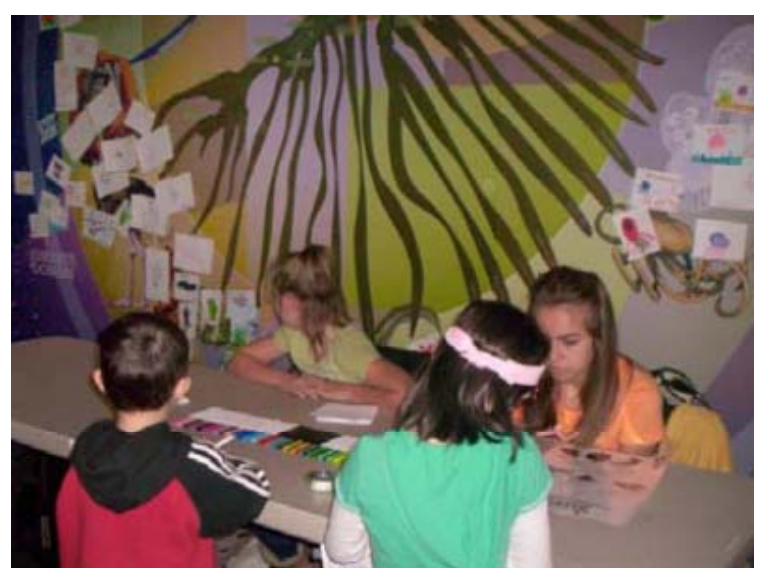

Figure 4. Volunteer-led activity at the Pittsburgh Zoo \& PPG Aquarium.

The next stage of our study will bring poster-sized representations of the murals into the classroom to assess their effectiveness as a classroom tool when paired with a teacher's guide and student activity booklets.

\section{Conclusion}

Our results demonstrate that some new understandings about the fundamental principles of evolution are being taught to patrons, but also point to ways that we can improve both the mural interaction and our evaluation methods.

We believe that the creation and placement of scientifically accurate art in public spaces and museums is a powerful tool for informal science education that can complement a variety of distinct learning styles. These same pieces can also be used in formal education as teaching tools that bridge traditional textbook materials.

When it comes to evolution, visualization is part of the research - it is used in data collection, interpretation, and communication. As such, art and visualization can and must contribute to the education of this topic, both in support of known information and by revealing the mental image that supports the hypothesis. In this way, we feel that visual literacy can significantly strengthen scientific literacy. We also found that images created with scientific depth can be used to tell different specific stories for a wide range of distinct audiences from young children, to informed teens, college students, 
and even professionals. The key is establishing the balance between the familiar and the new.

We propose that unsupervised surveys using computer touch screens have enormous potential in the evaluation of this type of educational experience. The method requires further study to increase the awareness of the museum visitors, the efficiency of the questions, and to decrease random answers.

\section{Acknowledgements}

Funded by the Regenerative Medicine Partnership in Education, a Science Education Partnership Award from the National Center for Research Resources (NIH). Additional research by Brinley Kantorski, Allison Pogue. Additional art by Robert Hoggard and Laura Gonzalez. Scientific advisors: Dr. Brady Porter, Dr. John Stolz, Dr. David Lampe, Dr. Michael Seaman of Biological Sciences, Duquesne University, and Dr. Matthew Lamanna, Ph.D. Carnegie Museum of Natural History. Thank you to the Children's Museum of Pittsburgh; the National Aviary of Pittsburgh; the Pittsburgh Zoo \& PPG Aquariums; the Phipps Conservatory, and the Carnegie Science Center.

\section{References}

[1] Newport F., On Darwin's Birthday, Only 4 in 10 Believe in Education, retrieved from http://www.gallup.com/poll/114544/Darwin-Birthday-

Believe-Evolution.aspx., access date: Feb 2009.

[2] van Wyhe J., The complete works of Charles Darwin online, retrieved from http://darwinonline.org.uk, access date 2009.

[3] Tree of Life Web Project, retrieved from http://tolweb.org, access date 2010.

[4] Interactive Tree of Life, retrieved from Itol.embl.de, Access date 2010.

[5] Ricou J., Pollock, J. A., Kantorski, B., Pogue, A., Bibliography for the Spiral of Life, retrieved from http://www.sepa.duq.edu/darwin/pdf/Sources_Spiral_Life. pdf, access date 2010.

[6] Ricou, J., Pollock, J. A., The Spiral of Life Mural Series, retrieved from http://www.sepa.duq.edu/darwin/ muralseries.shtml, access date 2010.

[7] Olsen, G.J., Woese, C.R., Overbeek, R., "The Winds of (Evolutionary) Change: Breathing New Life into Microbiology”, Journal of bacteriology, Jan. 1994, p. 1-6, Vol. 176, no.1.

[8] MacDonald, T., “Communicating Phylogeny: Evolutionary Tree Diagrams in Museums”, 2010 annual meeting of the National Association for Research in Science Teaching, Philadelphia, PA.
[9] Hand-out for Spiral of Life II: Plant and Animal Coevolution, retrieved from

http://store.greeneyevisualization.com/freedownload/Spiral OfLifeIIHandOutGEV.pdf. 